\title{
Distributional Analysis*
}

\author{
Tim Roughgarden ${ }^{\dagger}$
}

July 28,2020

\begin{abstract}
In distributional or average-case analysis, the goal is to design an algorithm with good-onaverage performance with respect to a specific probability distribution. Distributional analysis can be useful for the study of general-purpose algorithms on "non-pathological" inputs, and for the design of specialized algorithms in applications in which there is detailed understanding of the relevant input distribution. For some problems, however, pure distributional analysis encourages "overfitting" an algorithmic solution to a particular distributional assumption and a more robust analysis framework is called for. This chapter presents numerous examples of the pros and cons of distributional analysis, highlighting some of its greatest hits while also setting the stage for the hybrids of worst- and average-case analysis studied in later chapters.
\end{abstract}

\section{Introduction}

Part I of this book covered refinements of worst-case analysis which do not impose any assumptions on the possible inputs. Part II described several deterministic models of data, in which inputs to a problem were restricted to those with properties that are plausibly shared by all "real-world" inputs. This chapter, and a majority of the remaining chapters in the book, consider models that include a probability distribution over inputs.

\subsection{The Pros and Cons of Distributional Analysis}

In its purest form, the goal in distributional analysis is to analyze the average performance of algorithms with respect to a specific input distribution, and perhaps also to design new algorithms that perform particularly well for this distribution. What do we hope to gain from such an analysis?

- In applications in which the input distribution is well understood (e.g., due to lots of recent and representative data), distributional analysis is well suited both to predict the performance of existing algorithms and to design algorithms specialized to the input distribution.

- When there is a large gap between the empirical and worst-case performance of an algorithm, an input distribution can serve as a metaphor for "non-pathological" inputs. Even if the input distribution cannot be taken literally, a good average-case bound is a plausibility argument for the algorithm's empirical performance. The three examples in Section 2 are in this spirit.

\footnotetext{
${ }^{*}$ Chapter 8 of the book Beyond the Worst-Case Analysis of Algorithms (Roughgarden, 2020).

${ }^{\dagger}$ Department of Computer Science, Columbia University. Supported in part by NSF award CCF-1813188 and ARO award W911NF1910294. Email: tim.roughgarden@gmail.com.
} 
- Optimizing performance with respect to a specific input distribution can lead to new algorithmic ideas that are useful much more broadly. The examples in Sections 3 and 4 have this flavor.

And what could go wrong?

- Carrying out an average-case analysis of an algorithm might be analytically tractable only for the simplest (and not necessarily realistic) input distributions.

- Optimizing performance with respect to a specific input distribution can lead to "overfitting," meaning algorithmic solutions that are overly reliant on the details of the distributional assumptions and have brittle performance guarantees (which may not hold if the distributional assumptions are violated).

- Pursuing distribution-specific optimizations can distract from the pursuit of more robust and broadly useful algorithmic ideas.

This chapter has two goals. The first is to celebrate a few classical results in the average-case analysis of algorithms, which constitute some of the earliest work on alternatives to worst-case analysis. Our coverage here is far from encyclopedic, with the discussion confined to a sampling of relatively simple results for well-known problems that contribute to the chapter's overarching narrative. The second goal is to examine critically such average-case results, thereby motivating the more robust models of distributional analysis outlined in Section 5 and studied in detail later in the book.

\subsection{An Optimal Stopping Problem}

The pros and cons of distributional analysis are evident in a famous example from optimal stopping theory, which is interesting in its own right and also relevant to some of the random-order models described in Chapter 11. Consider a game with $n$ stages. Nonnegative prizes arrive online, with $v_{i}$ denoting the value of the prize that appears in stage $i$. At each stage, an algorithm must decide between accepting the current prize (which terminates the game) and proceeding to the next stage after discarding it. This involves a difficult trade-off, between the risk of being too ambitious (and skipping over what turns out to be the highest-value prize) and not ambitious enough (settling for a modest-value prize instead of waiting for a better one).

Suppose we posit specific distributions $D_{1}, D_{2}, \ldots, D_{n}$, known in advance to the algorithm designer, such that the value $v_{i}$ of the stage- $i$ prize is drawn independently from $D_{i}$. (The $D_{i}$ 's may or may not be identical.) An algorithm learns the realization $v_{i}$ of a prize value only at stage $i$. We can then speak about an optimal algorithm for the problem, meaning an online algorithm that achieves the maximum-possible expected prize value, where the expectation is with respect to the assumed distributions $D_{1}, D_{2}, \ldots, D_{n}$.

The optimal algorithm for a given sequence of prize value distributions is easy enough to specify, by working backward in time. If an algorithm finds itself at stage $n$ without having accepted a prize, it should definitely accept the final prize. (Recall all prizes have nonnegative values.) At an earlier stage $i$, the algorithm should accept the stage- $i$ prize if and only if $v_{i}$ is at least the expected prize value obtained by the (inductively defined) optimal strategy for stages $i+1, i+2, \ldots, n$.

\subsection{Discussion}

The solution above illustrates the primary advantages of distributional analysis: an unequivocal definition of an "optimal" algorithm, and the possibility of a crisp characterization of such an 
algorithm (as a function of the input distributions).

The disadvantages of average-case analysis are also on display, and there are several reasons why one might reject this optimal algorithm.

1. The algorithm takes the distributional assumptions literally and its description depends in a detailed way on the assumed distributions. It is unclear how robust the optimality guarantee is to misspecifications of these distributions, or to a reordering of the distributions.

2. The algorithm is relatively complicated, in that it is defined by $n$ different parameters (one threshold for each stage).

3. The algorithm does not provide any qualitative advice about how to tackle similar problems (other than "work backwards").

The third point is particularly relevant when studying a problem chosen as a deliberate simplification of a "real-world" problem that is too messy to analyze directly. In this case, an optimal solution to the simpler problem is useful only inasmuch as it suggests a plausibly effective solution to the more general problem.

For our optimal stopping problem, could there be non-trivial guarantees for simpler, more intuitive, and more robust algorithms?

\subsection{Threshold Rules and the Prophet Inequality}

Returning to the optimal stopping problem of Section 1.2, a threshold stopping rule is defined by a single parameter, a threshold $t$. The corresponding online algorithm accepts the first prize $i$ with value satisfying $v_{i} \geq t$ (if any). Such a rule is clearly suboptimal, as it doesn't even necessarily accept the prize at stage $n$. Nevertheless, the following prophet inequality proves that there is a threshold strategy with an intuitive threshold that performs approximately as well as a fully clairvoyant prophet. ${ }^{1}$

Theorem 1.1 (Samuel-Cahn (1984)). For every sequence $\mathbf{D}=D_{1}, D_{2}, \ldots, D_{n}$ of independent prize value distributions, there is a threshold rule that guarantees expected reward at least $\frac{1}{2} \mathbf{E}_{\mathbf{v} \sim \mathbf{D}}\left[\max _{i} v_{i}\right]$, where $\mathbf{v}$ denotes $\left(v_{1}, \ldots, v_{n}\right)$.

This guarantee holds, in particular, for the threshold $t$ at which there is a 50/50 chance that the rule accepts one of the $n$ prizes.

Proof. Let $z^{+}$denote $\max \{z, 0\}$. Consider a threshold strategy with threshold $t$ (to be chosen later). The plan is to prove a lower bound on the expected value of this strategy and an upper bound on the expected value of a prophet such that the two bounds are easy to compare.

What value does the $t$-threshold strategy obtain? Let $q(t)$ denote the probability of the failure mode where the threshold strategy accepts no prize at all; in this case, it obtains zero value. With the remaining probability $1-q(t)$, the rule obtains value at least $t$. To improve this lower bound, consider the case in which exactly one prize $i$ satisfies $v_{i} \geq t$; then, the rule also gets "extra credit" of $v_{i}-t$ above and beyond its baseline value of $t .^{2}$

\footnotetext{
${ }^{1}$ See Chapter 11 for an analogous result for a related problem, the secretary problem.

${ }^{2}$ The difficulty when two prizes $i$ and $j$ exceed the threshold is that this extra credit is either $v_{i}-t$ or $v_{j}-t$ (whichever appeared earlier). The proof avoids reasoning about the ordering of the distributions by crediting the rule only with the baseline value of $t$ in this case.
} 
Formally, we can bound the expected value obtained by the $t$-threshold strategy from below by

$$
\begin{aligned}
& (1-q(t)) \cdot t+ \\
& \sum_{i=1}^{n} \mathbf{E}_{\mathbf{v}}\left[v_{i}-t \mid v_{i} \geq t, v_{j}<t \forall j \neq i\right] \cdot \operatorname{Pr}\left[v_{i} \geq t\right] \cdot \operatorname{Pr}\left[v_{j}<t \forall j \neq i\right] \\
= & (1-q(t)) \cdot t+\sum_{i=1}^{n} \underbrace{\mathbf{E}_{\mathbf{v}}\left[v_{i}-t \mid v_{i} \geq t\right] \cdot \mathbf{P r}\left[v_{i} \geq t\right]}_{=\mathbf{E}\left[\left(v_{i}-t\right)^{+}\right]} \cdot \underbrace{\operatorname{Pr}\left[v_{j}<t \forall j \neq i\right]}_{\geq q(t)} \\
\geq & (1-q(t)) \cdot t+q(t) \sum_{i=1}^{n} \mathbf{E}_{\mathbf{v}}\left[\left(v_{i}-t\right)^{+}\right],
\end{aligned}
$$

where we use the independence of the $D_{i}$ 's in (1) to factor the two probability terms and in (2) to drop the conditioning on the event that $v_{j}<t$ for every $j \neq i$. In $(3)$, we use that $q(t)=$ $\operatorname{Pr}\left[v_{j}<t \forall j\right] \leq \operatorname{Pr}\left[v_{j}<t \forall j \neq i\right]$.

Now we produce an upper bound on the prophet's expected value $\mathbf{E}_{\mathbf{v} \sim \mathbf{D}}\left[\max _{i} v_{i}\right]$ that is easy to compare to (3). The expression $\mathbf{E}_{\mathbf{v}}\left[\max _{i} v_{i}\right]$ doesn't reference the strategy's threshold $t$, so we add and subtract it to derive

$$
\begin{aligned}
\mathbf{E}_{\mathbf{v}}\left[\max _{i=1}^{n} v_{i}\right] & =\mathbf{E}_{\mathbf{v}}\left[t+\max _{i=1}^{n}\left(v_{i}-t\right)\right] \\
& \leq t+\mathbf{E}_{\mathbf{v}}\left[\max _{i=1}^{n}\left(v_{i}-t\right)^{+}\right] \\
& \leq t+\sum_{i=1}^{n} \mathbf{E}_{\mathbf{v}}\left[\left(v_{i}-t\right)^{+}\right]
\end{aligned}
$$

Comparing (3) and (4), we can complete the proof by setting $t$ so that $q(t)=\frac{1}{2}$, with a $50 / 50$ chance of accepting a prize. ${ }^{3}$

The drawback of this threshold rule relative to the optimal online algorithm is clear: it does not guarantee as much expected value. Nonetheless, this solution possesses several attractive properties that are not satisfied by the optimal algorithm:

1. The threshold rule recommended by Theorem 1.1 depends on the prize value distributions $D_{1}, D_{2}, \ldots, D_{n}$ only inasmuch as it depends on the number $t$ for which there is a $50 / 50$ probability that at least one realized value exceeds $t$. For example, reordering the distributions arbitrarily does not change the recommended threshold rule.

2. A threshold rule is simple in that it is defined by only one parameter. Intuitively, a singleparameter rule is less prone to "overfitting" to the assumed distributions than a more highly parameterized algorithm like the ( $n$-parameter) optimal algorithm. ${ }^{4}$

3. Theorem 1.1 gives flexible qualitative advice about how to approach such problems: Start with threshold rules, and don't be too risk-averse (i.e., choose an ambitious enough threshold that receiving no prize is a distinct possibility).

\footnotetext{
${ }^{3}$ If there is no such $t$ because of point masses in the $D_{i}$ 's, then a minor extension of the argument yields the same result (Exercise 1).

${ }^{4}$ See Chapter 29 on data-driven algorithm design for a formalization of this intuition.
} 


\section{Average-Case Justifications of Classical Algorithms}

Distributional assumptions can guide the design of algorithms, as with the optimal stopping problem introduced in Section 1.2. Distributional analysis can also be used to analyze a general-purpose algorithm, with the goal of explaining mathematically why its empirical performance is much better than its worst-case performance. In these applications, the assumed probability distribution over inputs should not be taken literally; rather, it serves as a metaphor for "real-world" or "nonpathological" inputs. This section gives the flavor of work along these lines by describing one result for each of three classical problems: sorting, hashing, and bin packing.

\subsection{QuickSort}

Recall the QuickSort algorithm from undergraduate algorithms which, given an array of $n$ elements from a totally ordered set, works as follows:

- Designate one the $n$ array entries as a "pivot" element.

- Partition the input array around the pivot element $p$, meaning rearrange the array entries so that all entries less than $p$ appear before $p$ in the array and all entries greater than $p$ appear after $p$ in the array.

- Recursively sort the subarray comprising the elements less than $p$.

- Recursively sort the subarray comprising the elements greater than $p$.

The second step of the algorithm is easy to implement in $\Theta(n)$ time. There are many ways to choose the pivot element, and the running time of the algorithm varies between $\Theta(n \log n)$ and $\Theta\left(n^{2}\right)$, depending on these choices. ${ }^{5}$ One way to enforce the best-case scenario is to explicitly compute the median element and use it as the pivot. A simpler and more practical solution is to choose the pivot element uniformly at random; most of the time, it will be close enough to the median that both recursive calls are on significantly smaller inputs. A still simpler solution, which is common in practice, is to always use the first array element as the pivot element. This deterministic version of QuickSort runs in $\Theta\left(n^{2}\right)$ time on already-sorted arrays, but empirically its running time is $\Theta(n \log n)$ on almost all other inputs. One way to formalize this observation is to analyze the algorithm's expected running time on a random input. As a comparison-based sorting algorithm, the running time of QuickSort depends only on the relative order of the array entries, so we can assume without loss of generality that the input is a permutation of $\{1,2, \ldots, n\}$ and identify a "random input" with a random permutation. With any of the standard implementations of the partitioning subroutine, the average-case running time of this deterministic QuickSort algorithm is at most a constant factor larger than its best-case running time.

Theorem 2.1 (Hoare (1962)). The expected running time of the deterministic QuickSort algorithm on a random permutation of $\{1,2, \ldots, n\}$ is $O(n \log n)$.

Proof. We sketch one of the standard proofs. Assume that the partitioning subroutine only makes comparisons that involve the pivot element; this is the case for all of the textbook implementations. Each recursive call is given a subarray consisting of the elements from some interval $\{i, i+1, \ldots, j\}$; conditioned on this interval, the relative order of its elements in the subarray is uniformly random.

\footnotetext{
${ }^{5}$ In the best-case scenario, every pivot element is the median element of the subarray, leading to the recurrence $T(n)=2 T\left(\frac{n}{2}\right)+\Theta(n)$ with solution $\Theta(n \log n)$. In the worst-case scenario, every pivot element is the minimum or maximum element of the subarray, leading to the recurrence $T(n)=T(n-1)+\Theta(n)$ with solution $\Theta\left(n^{2}\right)$.
} 
Fix elements $i$ and $j$ with $i<j$. These elements are passed to the same sequence of recursive calls (along with $i+1, i+2, \ldots, j-1$ ), up to the first call in which an element from $\{i, i+1, \ldots, j\}$ is chosen as a pivot element. At this point, $i$ and $j$ are either compared to each other (if $i$ or $j$ was the chosen pivot) or not (otherwise); in any case, they are never compared to each other again in the future. With all subarray orderings equally likely, the probability that $i$ and $j$ are compared is exactly $\frac{2}{j-i+1}$. By the linearity of expectation, the expected total number of comparisons is then $\sum_{i=1}^{n-1} \sum_{j=i+1}^{n} \frac{2}{j-i+1}=O(n \log n)$, and the expected running time of the algorithm is at most a constant factor larger.

\subsection{Linear Probing}

A hash table is a data structure that supports fast insertions and lookups. Under the hood, most hash table implementations maintain an array $A$ of some length $n$ and use a hash function $h$ to map each inserted object $x$ to an array entry $h(x) \in\{1,2, \ldots, n\}$. A fundamental issue in hash table design is how to resolve collisions, meaning pairs $x, y$ of distinct inserted objects for which $h(x)=h(y)$. Linear probing is a specific way of resolving collisions:

1. Initially, all entries of $A$ are empty.

2. Store a newly inserted object $x$ in the first empty entry in the sequence $A[h(x)], A[h(x)+$ $1], A[h(x)+2], \ldots$, wrapping around to the beginning of the array, if necessary.

3. To search for an object $x$, scan the entries $A[h(x)], A[h(x)+1], A[h(x)+2], \ldots$ until encountering $x$ (a successful search) or an empty slot (an unsuccessful search), wrapping around to the beginning of the array, if necessary.

That is, the hash function indicates the starting position for an insertion or lookup operation, and the operation scans to the right until it finds the desired object or an empty position. The running time of an insertion or lookup operation is proportional to the length of this scan.

The bigger the fraction $\alpha$ of the hash table that is occupied (called its load), the fewer empty array entries and the longer the scans. To get calibrated, imagine searching for an empty array entry using independent and uniformly random probes. The number of attempts until a success is then a geometric random variable with success probability $1-\alpha$, which has expected value $\frac{1}{1-\alpha}$. With linear probing, however, objects tend to clump together in consecutive slots, resulting in slower operation times. How much slower?

Non-trivial mathematical guarantees for hash tables are possible only under assumptions that rule out data sets that are pathologically correlated with the table's hash function; for this reason, hash tables have long constituted one of the killer applications of average-case analysis. Common assumptions include asserting some amount of randomness in the data (as in average-case analysis), in the choice of hash function (as in randomized algorithms), or both (as in Chapter 26). For example, assuming that the data and hash function are such that every hash value $h(x)$ is an independent and uniform draw from $\{1,2, \ldots, n\}$, the expected time of insertions and lookups scales with $\frac{1}{(1-\alpha)^{2}} \cdot 6$

\footnotetext{
${ }^{6}$ This result played an important role in the genesis of the mathematical analysis of algorithms. Donald E. Knuth, its discoverer, wrote: "I first formulated the following derivation in 1962... Ever since that day, the analysis of algorithms has in fact been one of the major themes in my life."
} 


\subsection{Bin Packing}

The bin packing problem played a central role in the early development of the average-case analysis of algorithms; this section presents one representative result. ${ }^{7}$ Here, the average-case analysis is of the solution quality output by a heuristic (as with the prophet inequality), not its running time (unlike our QuickSort and linear probing examples).

In the bin packing problem, the input is $n$ items with sizes $s_{1}, s_{2}, \ldots, s_{n} \in[0,1]$. Feasible solutions correspond to ways of partitioning the items into bins so that the sum of the sizes in each bin is at most 1 . The objective is to minimize the number of bins used. This problem is NPhard, so every polynomial-time algorithm produces suboptimal solutions in some cases (assuming $P \neq N P)$.

Many practical bin packing heuristics have been studied extensively from both worst-case and average-case viewpoints. One example is the first-fit decreasing (FFD) algorithm:

- Sort and reindex the items so that $s_{1} \geq s_{2} \geq \cdots s_{n}$.

- For $i=1,2, \ldots, n$ :

- If there is an existing bin with room for item $i$ (i.e., with current total size at most $1-s_{i}$ ), add $i$ to the first such bin.

- Otherwise, start a new bin and add $i$ to it.

For example, consider an input consisting of 6 items with size $\frac{1}{2}+\epsilon, 6$ items with size $\frac{1}{4}+2 \epsilon, 6$ jobs with size $\frac{1}{4}+\epsilon$, and 12 items with size $\frac{1}{4}-2 \epsilon$. The FFD algorithm uses 11 bins while an optimal solution packs them perfectly into 9 bins (Exercise 3). Duplicating this set of 30 jobs as many times as necessary shows that there are arbitrarily large inputs for which the FFD algorithm uses $\frac{11}{9}$ times as many bins as an optimal solution. Conversely, the FFD algorithm never uses more than $\frac{11}{9}$ times the minimum-possible number of bins plus an additive constant (see the Notes for details).

The factor of $\frac{11}{9} \approx 1.22$ is quite good as worst-case approximation ratios go, but empirically the FFD algorithm usually produces a solution that is extremely close to optimal. One approach to better theoretical bounds is distributional analysis. For bin-packing algorithms, the natural starting point is the case in which item sizes are independent draws from the uniform distribution on $[0,1]$. Under this (strong) assumption, the FFD algorithm is near-optimal in a strong sense.

Theorem 2.2 (Frederickson (1980)). For every $\epsilon>0$, for $n$ items with sizes distributed independently and uniformly in [0,1], with probability $1-o(1)$ as $n \rightarrow \infty$, the FFD algorithm uses less than $(1+\epsilon)$ times as many bins as an optimal solution.

In other words, the typical approximation ratio of the FFD algorithm tends to 1 as the input size grows large.

We outline a two-step proof of Theorem 2.2. The first step shows that the guarantee holds for a less natural algorithm that we call the truncate and match (TM) algorithm. The second step shows that the FFD algorithm never uses more bins than the TM algorithm.

The truncate and match algorithm works as follows:

- Pack every item with size at least $1-\frac{2}{n^{1 / 4}}$ in its own bin. ${ }^{8}$

\footnotetext{
${ }^{7}$ See Chapter 11 for an analysis of bin packing heuristics in random-order models.

${ }^{8}$ For clarity, we omit ceilings and floors. See Exercise 4 for the motivation behind this size cutoff.
} 
- Sort and reindex the remaining $k$ items so that $s_{1} \geq s_{2} \geq \cdots \geq s_{k}$. (Assume for simplicity that $k$ is even.)

- For each $i=1, \ldots, k / 2$, put items $i$ and $k-i+1$ into a common bin if possible; otherwise, put them in separate bins.

To explain the intuition behind the TM algorithm, consider the expected order statistics (i.e., expected minimum, expected second-minimum, etc.) of $n$ independent samples from the uniform distribution on $[0,1]$. It can be shown that these split $[0,1]$ evenly into $n+1$ subintervals; the expected minimum is $\frac{1}{n+1}$, the expected second-minimum $\frac{2}{n+1}$, and so on. Thus at least in an expected sense, the first and last items together should fill up a bin exactly, as should the second and second-to-last items, and so on. Moreover, as $n$ grows large, the difference between the realized order statistics and their expectations should become small. Setting aside a small number of the largest items in the first step then corrects for any (small) deviations from these expectations with negligible additional cost. See Exercise 4 for details.

We leave the second step of the proof of Theorem 2.2 as Exercise 5 .

Lemma 2.1. For every bin packing input, the FFD algorithm uses at most as many bins as the TM algorithm.

The description of the general-purpose FFD algorithm is not tailored to a distributional assumption, but the proof of Theorem 2.2 is fairly specific to uniform-type distributions. This is emblematic of one of the drawbacks of average-case analysis: Often, it is analytically tractable only under quite specific distributional assumptions.

\section{Good-on-Average Algorithms for Euclidean Problems}

Another classical application domain for average-case analysis is in computational geometry, with the input comprising random points from some subset of Euclidean space. We highlight two representative results for fundamental problems in two dimensions, one concerning the running time of an always-correct convex hull algorithm and one about the solution quality of an efficient heuristic for the NP-hard Traveling Salesman Problem.

\section{$3.1 \quad 2 \mathrm{D}$ Convex Hull}

A typical textbook on computational geometry begins with the $2 D$ convex hull problem. The input consists of a set $S$ of $n$ points in the plane (in the unit square [0,1] $\times[0,1]$, say) and the goal is to report, in sorted order, the points of $S$ that lie on the convex hull of $S .{ }^{9}$ There are several algorithms that solve the $2 \mathrm{D}$ convex hull problem in $\Theta(n \log n)$ time. Can we do better-perhaps even linear time - when the points are drawn from a distribution, such as the uniform distribution on the square?

Theorem 3.1 (Bentley and Shamos (1978)). There is an algorithm that solves the 2D convex hull problem in expected $O(n)$ time for $n$ points drawn independently and uniformly from the unit square.

The algorithm is a simple divide-and-conquer algorithm. Given points $S=\left\{p_{1}, p_{2}, \ldots, p_{n}\right\}$ drawn independently and uniformly from the plane:

\footnotetext{
${ }^{9}$ Recall that the convex hull of a set of points is the smallest convex set containing them, or equivalently the set of all convex combinations of points from $S$. In two dimensions, imagine the points as nails in a board, and the convex hull as a taut rubber band that encloses them.
} 
- If the input $S$ contains at most 5 points, compute the convex hull by brute force. Return the points of $S$ on the convex hull, sorted by $x$-coordinate.

- Otherwise, let $S_{1}=\left\{p_{1}, \ldots, p_{n / 2}\right\}$ and $S_{2}=\left\{p_{(n / 2)+1}, \ldots, p_{n}\right\}$ denote the first and second halves of $S$. (Assume for simplicity that $n$ is even.)

- Recursively compute the convex hull $C_{1}$ of $S_{1}$, with its points sorted by $x$-coordinate.

- Recursively compute the convex hull $C_{2}$ of $S_{2}$, with its points sorted by $x$-coordinate.

- Merge $C_{1}$ and $C_{2}$ into the convex hull $C$ of $S$. Return $C$, with the points of $C$ sorted by $x$-coordinate.

For every set $S$ and partition of $S$ into $S_{1}$ and $S_{2}$, every point on the convex hull of $S$ is on the convex hull of either $S_{1}$ or $S_{2}$. Correctness of the algorithm follows immediately. The last step is easy to implement in time linear in $\left|C_{1}\right|+\left|C_{2}\right|$; see Exercise 6 . Because the subproblems $S_{1}$ and $S_{2}$ are themselves uniformly random points from the unit square (with the sorting occurring only after the recursive computation completes), the expected running time of the algorithm is governed by the recurrence

$$
T(n) \leq 2 \cdot T\left(\frac{n}{2}\right)+O\left(\mathbf{E}\left[\left|C_{1}\right|+\left|C_{2}\right|\right]\right) .
$$

Theorem 3.1 follows immediately from this recurrence and the following combinatorial bound.

Lemma 3.1 (Rényi and Sulanke (1963)). The expected size of the convex hull of $n$ points drawn independently and uniformly from the unit square is $O(\log n)$.

Proof. Imagine drawing the input points in two phases, with $\frac{n}{2}$ points $S_{i}$ drawn in phase $i$ for $i=1,2$. An elementary argument shows that the convex hull of the points in $S_{1}$ occupies, in expectation, at least a $1-O\left(\frac{\log n}{n}\right)$ fraction of the unit square (Exercise 7 ). Each point of the second phase thus lies in the interior of the convex hull of $S_{1}$ (and hence of $S_{1} \cup S_{2}$ ) except with probability $O\left(\frac{\log n}{n}\right)$, so the expected number of points from $S_{2}$ on the convex hull of $S_{1} \cup S_{2}$ is $O(\log n)$. By symmetry, the same is true of $S_{1}$.

\subsection{The Traveling Salesman Problem in the Plane}

In the Traveling Salesman Problem (TSP), the input consists of $n$ points and distances between them, and the goal is to compute a tour of the points (visiting each point once and returning to the starting point) with the minimum-possible total length. In Euclidean TSP, the points lie in Euclidean space and all distances are straight-line distances. This problem is $N P$-hard, even in two dimensions. The main result of this section is analogous to Theorem 2.2 in Section 2.3 for the bin packing problem - a polynomial-time algorithm that, when the input points are drawn independently and uniformly from the unit square, has approximation ratio tending to 1 (with high probability) as $n$ tends to infinity.

The algorithm, which we call the Stitch algorithm, works as follows:

- Divide the unit square evenly into $s=\frac{n}{\ln n}$ subsquares, each with side length $\sqrt{(\ln n) / n} \cdot{ }^{10}$

- For each subsquare $i=1,2, \ldots, s$, containing the points $P_{i}$ :

\footnotetext{
${ }^{10}$ Again, we ignore ceilings and floors.
} 
- If $\left|P_{i}\right| \leq 6 \log _{2} n$, compute the optimal tour $T_{i}$ of $P_{i}$ using dynamic programming. ${ }^{11}$

- Otherwise, return an arbitrary tour $T_{i}$ of $P_{i}$.

- Choose an arbitrary representative point from each non-empty set $P_{i}$, and let $R$ denote the set of representatives.

- Construct a tour $T_{0}$ of $R$ by visiting points from left-to-right in the bottommost row of subsquares, right-to-left in the second-to-bottom row, and so on, returning to the starting point after visiting all the points in the topmost row.

- Shortcut the union of the subtours $\cup_{i=0}^{s} T_{i}$ to a single tour $T$ of all $n$ points, and return $T .{ }^{12}$

This algorithm runs in polynomial time with probability 1 and returns a tour of the input points. As for the approximation guarantee:

Theorem 3.2 (Karp (1977)). For every $\epsilon>0$, for $n$ points distributed independently and uniformly in the unit square, with probability $1-o(1)$ as $n \rightarrow \infty$, the Stitch algorithm returns a tour with total length less than $(1+\epsilon)$ times that of an optimal tour.

Proving Theorem 3.2 requires understanding the typical length of an optimal tour of random points in the unit square and then bounding from above the difference between the lengths of the tour returned by the Stitch algorithm and of the optimal tour. The first step is not difficult (Exercise 8).

Lemma 3.2. There is a constant $c_{1}>0$ such that, with probability $1-o(1)$ as $n \rightarrow \infty$, the length of an optimal tour of $n$ points drawn independently and uniformly from the unit square is at least $c_{1} \sqrt{n}$.

Lemma 3.2 implies that proving Theorem 3.2 reduces to showing that (with high probability) the difference between the lengths of Stitch's tour and the optimal tour is $o(\sqrt{n})$.

For the second step, we start with a simple consequence of the Chernoff bound (see Exercise 9 ).

Lemma 3.3. In the Stitch algorithm, with probability $1-o(1)$ as $n \rightarrow \infty$, every subsquare contains at most $6 \log _{2} n$ points.

It is also easy to bound the length of the tour $T_{0}$ of the representative points $R$ in the Stitch algorithm (see Exercise 10).

Lemma 3.4. There is a constant $c_{2}$ such that, for every input, the length of the tour $T_{0}$ in the Stitch algorithm is at most

$$
c_{2} \cdot \sqrt{s}=c_{2} \cdot \sqrt{\frac{n}{\ln n}} .
$$

The key lemma states that an optimal tour can be massaged into subtours for all of the subsquares without much additional cost.

\footnotetext{
${ }^{11}$ Given $k$ points, label them $\{1,2, \ldots, k\}$. There is one subproblem for each subset $S$ of points and point $j \in S$, whose solution is the minimum-length path that starts at the point 1 , ends at the point $j$, and visits every point of $S$ exactly once. Each of the $O\left(k 2^{k}\right)$ subproblems can be solved in $O(k)$ time by trying all possibilities for the final hop of the optimal path. When $k=O(\log n)$, this running time of $O\left(k^{2} 2^{k}\right)$ is polynomial in $n$.

${ }^{12}$ The union of the $s+1$ subtours can be viewed as a connected Eulerian graph, which then admits a closed Eulerian walk (using every edge of the graph exactly once). This walk can be transformed to a tour of the points with only smaller length by skipping repeated visits to a point.
} 
Lemma 3.5. Let $T^{*}$ denote an optimal tour of the $n$ input points, and let $L_{i}$ denote the length of the portion of $T^{*}$ that lies within the subsquare $i \in\{1,2, \ldots, s\}$ defined by the Stitch algorithm. For every subsquare $i=1,2, \ldots, s$, there exists a tour of the points $P_{i}$ in the subsquare of length at most

$$
L_{i}+6 \sqrt{\frac{\ln n}{n}} .
$$

The key point in Lemma 3.5 is that the upper bound in (5) depends only on the size of the square, and not on the number of times that the optimal tour $T^{*}$ crosses its boundaries.

Before proving Lemma 3.5, we observe that Lemmas 3.2-3.5 easily imply Theorem 3.2. Indeed, with high probability:

1. The optimal tour has length $L^{*} \geq c_{1} \sqrt{n}$.

2. Every subsquare in the Stitch algorithm contains at most $6 \ln n$ points, and hence the algorithm computes an optimal tour of the points in each subsquare (with length at most (5)).

3. Thus, recalling that $s=\frac{n}{\ln n}$, the total length of Stitch's tour is at most

$$
\sum_{i=1}^{s}\left(L_{i}+6 \sqrt{\frac{\ln n}{n}}\right)+c_{2} \cdot \sqrt{\frac{n}{\ln n}}=L^{*}+O\left(\sqrt{\frac{n}{\ln n}}\right)=(1+o(1)) \cdot L^{*} .
$$

Finally, we prove Lemma 3.5.

Proof. (Lemma 3.5) Fix a subsquare $i$ with a non-empty set $P_{i}$ of points. The optimal tour $T^{*}$ visits every point in $P_{i}$ while crossing the boundary of the subsquare an even number $2 t$ of times; denote these crossing points by $Q_{i}=\left\{y_{1}, y_{2}, \ldots, y_{2 t}\right\}$, indexed in clockwise order around the subsquare's perimeter (starting from the lower left corner). Now form a connected Eulerian multi-graph $G=$ $(V, E)$ with vertices $V=P_{i} \cup Q_{i}$ by adding the following edges:

- Add the portions of $T^{*}$ that lie inside the subsquare (giving points of $P_{i}$ a degree of 2 and points of $Q_{i}$ a degree of 1 ).

- Let $M_{1}$ (respectively, $M_{2}$ ) denote the perfect matching of $Q_{i}$ that matches each $y_{j}$ with $j$ odd (respectively, with $j$ even) to $y_{j+1}$. (In $M_{2}, y_{2 t}$ is matched with $y_{1}$.) Add two copies of the cheaper matching to the edge set $E$ and one copy of the more expensive matching (boosting the degree of points of $Q_{i}$ to 4 while also ensuring connectivity).

The total length of the edges contributed by the first ingredient is $L_{i}$. The total length of the edges in $M_{1} \cup M_{2}$ is at most the perimeter of the subsquare, which is $4 \sqrt{\frac{\ln n}{n}}$. The second copy of the cheaper matching adds at most $2 \sqrt{\frac{\ln n}{n}}$ to the total length of the edges in $G$. As in footnote 12 , because $G$ is connected and Eulerian, we can extract from it a tour of $P_{i} \cup Q_{i}$ (and hence of $P_{i}$ ) that has total length at most that of the edges of $G$, which is at most $L_{i}+6 \sqrt{\frac{\ln n}{n}}$.

\subsection{Discussion}

To what extent are the two divide-and-conquer algorithms of this section tailored to the distributional assumption that the input points are drawn independently and uniformly at random 
from the unit square? For the convex hull algorithm in Section 3.1, the consequence of an incorrect distributional assumption is mild; its worst-case running time is governed by the recurrence $T(n) \leq 2 T\left(\frac{n}{2}\right)+O(n)$ and hence is $O(n \log n)$, which is close to linear. Also, analogs of Lemma 3.1 (and hence Theorem 3.1) can be shown to hold for a number of other distributions.

The Stitch algorithm in Section 3.2, with its fixed dissection of the unit square into equal-size subsquares, may appear hopelessly tied to the assumption of a uniform distribution. But minor modifications to it result in more robust algorithms, for example by using an adaptive dissection, which recursively divides each square along either the median $x$-coordinate or the median $y$-coordinate of the points in the square. Indeed, this idea paved the way for later algorithms that obtained polynomial-time approximation schemes (i.e., $(1+\epsilon)$-approximations for arbitrarily small constant $\epsilon$ ) even for the worst-case version of Euclidean TSP (see the Notes).

Zooming out, our discussion of these two examples touches on one of the biggest risks of averagecase analysis: distributional assumptions can lead to algorithms that are unduly tailored to the assumptions. On the other hand, even when this is the case, the high-level ideas behind the algorithms can prove useful much more broadly.

\section{Random Graphs and Planted Models}

Most of our average-case models so far concern random numerical data. This section studies random combinatorial structures, and specifically different probability distributions over graphs.

\subsection{Erdős-Rényi Random Graphs}

This section reviews the most well-studied model of random graphs, the Erdös-Rényi random graph model. This model is a family $\left\{\mathcal{G}_{n, p}\right\}$ of distributions, indexed by the number $n$ of vertices and the edge density $p$. A sample from the distribution $\mathcal{G}_{n, p}$ is a graph $G=(V, E)$ with $|V|=n$ and each of the $\left(\begin{array}{l}n \\ 2\end{array}\right)$ possible edges present independently with probability $p$. The special case of $p=\frac{1}{2}$ is the uniform distribution over all $n$-vertex graphs. This is an example of an "oblivious random model," meaning that it is defined independently of any particular optimization problem.

The assumption of uniformly random data may have felt like cheating already in our previous examples, but it is particularly problematic for many computational problems on graphs. Not only is this distributional assumption extremely specific, it also fails to meaningfully differentiate between different algorithms. ${ }^{13}$ We illustrate this point with two problems that are discussed at length in Chapters 9 and 10.

Example: Minimum bisection. In the graph bisection problem, the input is an undirected graph $G=(V, E)$ with an even number of vertices, and the goal is to identify a bisection (i.e., a partition of $V$ into two equal-size groups) with the fewest number of crossing edges.

To see why this problem is algorithmically uninteresting in the Erdős-Rényi random graph model, take $p=\frac{1}{2}$ and let $n$ tend to infinity. In a random sample from $\mathcal{G}_{n, p}$, for every bisection $(S, \bar{S})$ of the set $V$ of $n$ vertices, the expected number of edges of $E$ crossing it is $\frac{n^{2}}{8}$. A straightforward application of the Chernoff bound shows that, with probability $1-o(1)$ as $n \rightarrow \infty$, the number of edges crossing every bisection is $(1 \pm o(1)) \cdot \frac{n^{2}}{8}$ (Exercise 11). Thus even an algorithm that computes a maximum bisection is an almost optimal algorithm for computing a minimum bisection!

\footnotetext{
${ }^{13}$ It also fails to replicate the statistical properties commonly observed in "real-world" graphs; see Chapter 28 for further discussion.
} 
Example: Maximum clique. In the maximum clique problem, the goal (given an undirected graph) is to identify the largest subset of vertices that are mutually adjacent. In a random graph in the $\mathcal{G}_{n, 1 / 2}$ model, the size of the maximum clique is very likely to be $\approx 2 \log _{2} n .{ }^{14}$ To see heuristically why this is true, note that for an integer $k$, the expected number of cliques on $k$ vertices in a random graph of $\mathcal{G}_{n, 1 / 2}$ is exactly

$$
\left(\begin{array}{l}
n \\
k
\end{array}\right) 2^{-\left(\begin{array}{l}
k \\
2
\end{array}\right)} \approx n^{k} 2^{-k^{2} / 2}
$$

which is 1 precisely when $k=2 \log _{2} n$. That is, $2 \log _{2} n$ is approximately the largest $k$ for which we expect to see at least one $k$-clique.

On the other hand, while there are several polynomial-time algorithms (including the obvious greedy algorithm) that compute, with high probability, a clique of $\operatorname{size} \approx \log _{2} n$ in a random graph from $\mathcal{G}_{n, 1 / 2}$, no such algorithm is known to do better. The Erdös-Rényi model fails to distinguish between different efficient heuristics for the Maximum Clique problem.

\subsection{Planted Graph Models}

Chapters 5 and 6 study deterministic models of data in which the optimal solution to an optimization problem must be "clearly optimal" in some sense, with the motivation of zeroing in on the instances with a "meaningful" solution (such as an informative clustering of data points). Planted graph models implement the same stability idea in the context of random graphs, by positing probability distributions over inputs which generate (with high probability) graphs in which an optimal solution "sticks out." The goal is then to devise a polynomial-time algorithm that recovers the optimal solution with high probability, under the weakest-possible assumptions on the input distribution. Unlike an oblivious random model such as the Erdős-Rényi model, planted models are generally defined with a particular computational problem in mind.

Algorithms for planted models generally fall into three categories, listed roughly in order of increasing complexity and power.

1. Combinatorial approaches. We leave the term "combinatorial" safely undefined, but basically it refers to algorithms that work directly with the graph, rather than resorting to any continuous methods. For example, an algorithm that looks only at vertex degrees, subgraphs, shortest paths, etc., would be considered combinatorial.

2. Spectral algorithms. Here "spectral" means an algorithm that computes and uses the eigenvectors of a suitable matrix derived from the input graph. Spectral algorithms often achieve optimal recovery guarantees for planted models.

3. Semidefinite programming (SDP). Algorithms that use semidefinite programming have proved useful for extending guarantees for spectral algorithms in planted models to hold also in semirandom models (see Chapters 9 and 10).

Example: Planted bisection. In the planted bisection problem, a graph is generated according to the following random process (for a fixed vertex set $V$, with $|V|$ even, and parameters $p, q \in[0,1]$ ):

1. Choose a partition $(S, T)$ of $V$ with $|S|=|T|$ uniformly at random.

2. Independently for each pair $(i, j)$ of vertices inside the same cluster $(S$ or $T)$, include the edge $(i, j)$ with probability $p$.

\footnotetext{
${ }^{14}$ In fact, the size of the maximum clique turns out to be incredibly concentrated; see the Notes.
} 
3. Independently for each pair $(i, j)$ of vertices in different clusters, include the edge $(i, j)$ with probability $q .{ }^{15}$

Thus the expected edge density inside the clusters is $p$, and between the clusters is $q$.

The difficulty of recovering the planted bisection $(S, T)$ clearly depends on the gap between $p$ and $q$. The problem is impossible if $p=q$ and trivial if $p=1$ and $q=0$. Thus the key question in this model is: how big does the gap $p-q$ need to be before exact recovery is possible in polynomial time (with high probability)?

When $p, q$, and $p-q$ are bounded below by a constant independent of $n$, the problem is easily solved by combinatorial approaches (Exercise 12); unfortunately, these do not resemble algorithms that perform well in practice.

We can make the problem more difficult by allowing $p, q$, and $p-q$ to go to 0 with $n$. Here, semidefinite programming-based algorithms work for an impressively wide range of parameter values. For example:

Theorem 4.1 (Abbe et al. (2016); Hajek et al. (2016)). If $p=\frac{\alpha \ln n}{n}$ and $q=\frac{\beta \ln n}{n}$ with $\alpha>\beta$, then:

(a) If $\sqrt{\alpha}-\sqrt{\beta} \geq \sqrt{2}$, there is a polynomial-time algorithm that recovers the planted partition $(S, T)$ with probability $1-o(1)$ as $n \rightarrow \infty$.

(b) If $\sqrt{\alpha}-\sqrt{\beta}<\sqrt{2}$, then no algorithm recovers the planted partition with constant probability as $n \rightarrow \infty$.

In this parameter regime, semidefinite programming algorithms provably achieve informationtheoretically optimal recovery guarantees. Thus, switching from the $p, q, p-q=\Omega(1)$ parameter regime to the $p, q, p-q=o(1)$ regime is valuable not because we literally believe that the latter is more faithful to "real-world" instances, but rather because it encourages better algorithm design.

Example: Planted clique. The planted clique problem with parameters $k$ and $n$ concerns the following distribution over graphs.

1. Fix a vertex set $V$ with $n$ vertices. Sample a graph from $\mathcal{G}_{n, 1 / 2}$ : Independently for each pair $(i, j)$ of vertices, include the edge $(i, j)$ with probability $\frac{1}{2}$.

2. Choose a random subset $Q \subseteq V$ of $k$ vertices.

3. Add all remaining edges between pairs of vertices in $Q$.

Once $k$ is significantly bigger than $\approx 2 \log _{2} n$, the likely size of a maximum clique in a random graph from $\mathcal{G}_{n, 1 / 2}$, the planted clique $Q$ is with high probability the maximum clique of the graph. How big does $k$ need to be before it becomes visible to a polynomial-time algorithm?

When $k=\Omega(\sqrt{n \log n})$, the problem is trivial, with the $k$ highest-degree vertices constituting the planted clique $Q$. To see why this is true, think first about the sampled Erdös-Rényi random graph, before the clique $Q$ is planted. The expected degree of each vertex is $\approx n / 2$, with standard deviation $\approx \sqrt{n} / 2$. Textbook large deviation inequalities show that, with high probability, the degree of every vertex is within $\approx \sqrt{\ln n}$ standard deviations of its expectation (Figure 1). Planting a clique $Q$ of size $a \sqrt{n \log n}$, for a sufficiently large constant $a$, then boosts the degrees of all of the clique vertices enough that they catapult past the degrees of all of the non-clique vertices.

\footnotetext{
${ }^{15}$ This model is a special case of the stochastic block model studied in Chapter 10.
} 


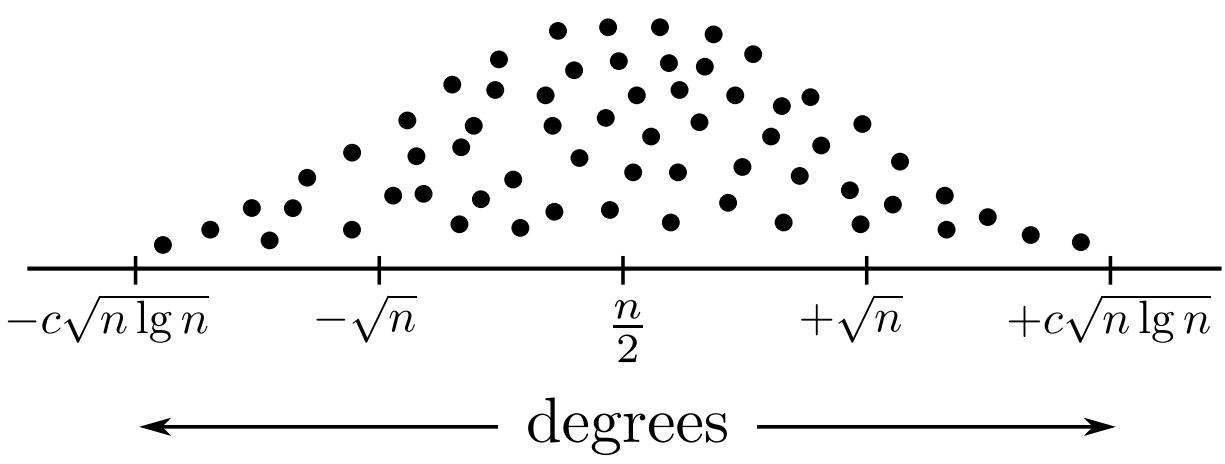

Figure 1: Degree distribution of an Erdős-Rényi random graph with edge density $\frac{1}{2}$, before planting the $k$-clique $Q$. If $k=\Omega(\sqrt{n \lg n})$, then the planted clique will consist of the $k$ vertices with the highest degrees.

The "highest degrees" algorithm is not very useful in practice. What went wrong? The same thing that often goes wrong with pure average-case analysis - the solution is brittle and overly tailored to a specific distributional assumption. How can we change the input model to encourage the design of algorithms with more robust guarantees?

One idea is to mimic what worked well for the planted bisection problem, and to study a more difficult parameter regime that forces us to develop more useful algorithms. For the planted clique problem, there are non-trivial algorithms, including spectral algorithms, that recover the planted clique $Q$ with high probability provided $k=\Omega(\sqrt{n})$ (see the Notes).

\subsection{Discussion}

There is a happy ending to the study of both the planted bisection and planted clique problems: with the right choice of parameter regimes, these models drive us toward non-trivial algorithms that might plausibly be useful starting points for the design of practical algorithms. Still, both results seem to emerge from "threading the needle" in the parameter space. Could there be a better alternative, in the form of input models that explicitly encourage the design of robustly good algorithms?

\section{Robust Distributional Analysis}

Many of the remaining chapters in this book pursue different hybrids of worst- and average-case analysis, in search of a "sweet spot" for algorithm analysis that both encourages robustly good algorithms (like in worst-case analysis) and allows for strong provable guarantees (like in averagecase analysis). Most of these models assume that there is in fact a probability distribution over inputs (as in average-case analysis), but that this distribution is a priori unknown to an algorithm. The goal is then to design algorithms that work well no matter what the input distribution is (perhaps with some restrictions on the class of possible distributions). Indeed, several of the average-case guarantees in this chapter can be viewed as applying simultaneously (i.e., in the worst case) across a restricted but still infinite family of input distributions:

- The $\frac{1}{2}$-approximation in the prophet inequality (Theorem 1.1) for a threshold- $t$ rule applies simultaneously to all distribution sequences $D_{1}, D_{2}, \ldots, D_{n}$ such that $\operatorname{Pr}_{\mathbf{v} \sim \mathbf{D}}\left[\max _{i} v_{i} \geq t\right]=\frac{1}{2}$ (e.g., all possible reorderings of one such sequence). 
- The guarantees for our algorithms for the bin packing (Theorem 2.2), convex hull (Theorem 3.1), and Euclidean TSP (Theorem 3.2) problems hold more generally for all input distributions that are sufficiently close to uniform.

The general research agenda in robust distributional analysis is to prove approximate optimality guarantees for algorithms for as many different computational problems and as rich a class of input distributions as possible. Work in the area can be divided into two categories, both well represented in this book, depending on whether an algorithm observes one or many samples from the unknown input distribution. We conclude this chapter with an overview of what's to come.

\subsection{Simultaneous Near-Optimality}

In single-sample models, an algorithm is designed with knowledge only of a class $\mathcal{D}$ of possible input distributions, and receives only a single input drawn from an unknown and adversarially chosen distribution from $\mathcal{D}$. In these models, the algorithm cannot hope to learn anything non-trivial about the input distribution. Instead, the goal is to design an algorithm that, for every input distribution $D \in \mathcal{D}$, has expected performance close to that of the optimal algorithm specifically tailored for $D$. Examples include:

- The semi-random models in Chapters 9-11 and 17 and the smoothed analysis models in Chapters 13-15 and 19. In these models, nature and an adversary collaborate to produce an input, and each fixed adversary strategy induces a particular input distribution. Performing well with respect to the adversary in these models is equivalent to performing well simultaneously across all of the induced input distributions.

- The effectiveness of simple hash functions with pseudorandom data (Chapter 26). The main result in this chapter is a guarantee for universal hashing that holds simultaneously across all data distributions with sufficient entropy.

- Prior-independent auctions (Chapter 27), which are auctions that achieve near-optimal expected revenue simultaneously across a wide class of valuation distributions.

\subsection{Learning a Near-Optimal Solution}

In multi-sample models, an algorithm observes multiple samples from an unknown input distribution $D \in \mathcal{D}$, and the goal is to efficiently identify a near-optimal algorithm for $D$ from as few samples as possible. Examples include:

- Self-improving algorithms (Chapter 12) and data-driven algorithm design (Chapter 29), in which the goal is to design an algorithm that, when presented with independent samples from an unknown input distribution, quickly converges to an approximately best-in-class algorithm for that distribution.

- Supervised learning (Chapters 16 and 22), in which the goal is to identify the expected lossminimizing hypothesis (from a given hypothesis class) for an unknown data distribution given samples from that distribution.

- Distribution testing (Chapter 23), in which the goal is to make accurate inferences about an unknown distribution from a limited number of samples. 


\section{Notes}

The prophet inequality (Theorem 1.1) is due to Samuel-Cahn (1984). The pros and cons of threshold rules versus optimal online algorithms are discussed also by Hartline (2017). QuickSort and its original analysis are due to Hoare $(1962)$. The $(1-\alpha)^{-2}$ bound for linear probing with load $\alpha$ and random data, as well as the corresponding quote in Section 2.2, are in Knuth (1998). A good (if outdated) entry point to the literature on bin packing is Coffman, Jr. et al. (1996). The lower bound for the FFD algorithm in Exercise 3 is from Johnson et al. (1974). The first upper bound of the form $\frac{11}{9} \cdot O P T+O(1)$ for the number of bins used by the FFD algorithm, where $O P T$ denotes the minimum-possible number of bins, is due to Johnson (1973). The exact worst-case bound for FFD was pinned down recently by Dósa et al. (2013). The average-case guarantee in Theorem 2.2 is a variation on one by Frederickson (1980), who proved that the expected difference between the number of bins used by FFD and an optimal solution is $O\left(n^{2 / 3}\right)$. A more sophisticated argument gives a tight bound of $\Theta\left(n^{1 / 2}\right)$ on this expectation (Coffman, Jr. et al., 1991).

The linear expected time algorithm for 2D convex hulls (Theorem 3.1) is by Bentley and Shamos (1978). Lemma 3.1 was first proved by Rényi and Sulanke (1963); the proof outlined here follows Har-Peled (1998). Exercise 6 is solved by Andrews (1979). The asymptotic optimality of the Stitch algorithm for Euclidean TSP (Theorem 3.2) is due to Karp (1977), who also gave an alternative solution based on the adaptive dissections mentioned in Section 3.3. A good general reference for this topic is Karp and Steele (1985). The worst-case approximation schemes mentioned in Section 3.3 are due to Arora (1998) and Mitchell (1999).

The Erdős-Rényi random graph model is from Erdős and Rényi (1960). The size of the maximum clique in a random graph drawn from $\mathcal{G}_{n, 1 / 2}$ was characterized by Matula (1976); with high probability it is either $k$ or $k+1$, where $k$ is an integer roughly equal to $2 \log _{2} n$. Grimmett and McDiarmid (1975) proved that the greedy algorithm finds, with high probability, a clique of size roughly $\log _{2} n$ in a random graph from $\mathcal{G}_{n, 1 / 2}$. The planted bisection model described here was proposed by Bui et al. (1987) and is also a special case of the stochastic block model defined by Holland et al. (1983). Part (b) of Theorem 4.1 and a weaker version of part (a) were proved by Abbe et al. (2016); the stated version of part (a) is due to Hajek et al. (2016). The planted clique model was suggested by Jerrum (1992). Kucera (1995) noted that the "top- $k$ degrees" algorithm works with high probability when $k=\Omega(\sqrt{n \log n})$. The first polynomial-time algorithm for the planted clique problem with $k=O(\sqrt{n})$ was the spectral algorithm of Alon et al. (1998). Barak et al. (2016) supplied evidence, in the form of a sum-of-squares lower bound, that the planted clique problem is intractable when $k=o(\sqrt{n})$.

The versions of the Chernoff bound stated in Exercises 4(a) and 9 can be found, for example, in Mitzenmacher and Upfal (2017).

\section{Acknowledgments}

I thank Anupam Gupta, C. Seshadhri, and Sahil Singla for helpful comments on a preliminary draft of this chapter.

\section{References}

Abbe, E., A. S. Bandeira, and G. Hall (2016). Exact recovery in the stochastic block model. IEEE Transactions on Information Theory 62(1), 471-487. 
Alon, N., M. Krivelevich, and B. Sudakov (1998). Finding a large hidden clique in a random graph. Random Structures 85 Algorithms 13(3-4), 457-466.

Andrews, A. M. (1979). Another efficient algorithm for convex hulls in two dimensions. Information Processing Letters 9(5), 216-219.

Arora, S. (1998). Polynomial time approximation schemes for euclidean traveling salesman and other geometric problems. Journal of the ACM 45(5), 753-782.

Barak, B., S. B. Hopkins, J. A. Kelner, P. Kothari, A. Moitra, and A. Potechin (2016). A nearly tight sum-of-squares lower bound for the planted clique problem. In Proceedings of the 57th Annual IEEE Symposium on Foundations of Computer Science (FOCS), pp. 428-437.

Bentley, J. L. and M. I. Shamos (1978). Divide and conquer for linear expected time. Information Processing Letters 7(2), 87-91.

Bui, T. N., S. Chaudhuri, F. T. Leighton, and M. Sipser (1987). Graph bisection algorithms with good average case behavior. Combinatorica 7(2), 171-191.

Coffman, Jr., E. G., C. Courcoubetis, M. R. Garey, D. S. Johnson, L. A. McGeoch, P. W. Shor, R. R. Weber, and M. Yannakakis (1991). Fundamental discrepancies between average-case analyses under discrete and continuous distributions: A bin packing case study. In Proceedings of the 23rd Annual ACM Symposium on Theory of Computing (STOC), pp. 230-240.

Coffman, Jr., E. G., M. R. Garey, and D. S. Johnson (1996). Approximation algorithms for bin packing: A survey. In D. Hochbaum (Ed.), Approximation Algorithms for NP-Hard Problems, Chapter 2, pp. 46-93. PWS.

Dósa, G., R. Li, X. Hanc, and Z. Tuza (2013). Tight absolute bound for first fit decreasing binpacking: $F F D(L) \leq 11 / 9 O P T(L)+6 / 9$. Theoretical Computer Science 510, 13-61.

Erdős, P. and A. Rényi (1960). On the evolution of random graphs. Publ. Math. Inst. Hungar. Acad. Sci. 5, 17-61.

Frederickson, G. N. (1980). Probabilistic analysis for simple one- and two-dimensional bin packing algorithms. Information Processing Letters 11(4-5), 156-161.

Grimmett, G. and C. J. H. McDiarmid (1975). On colouring random graphs. Mathematical Proceedings of the Cambridge Philosophical Society 77, 313-324.

Hajek, B., Y. Wu, and J. Xu (2016). Achieving exact cluster recovery threshold via semidefinite programming: Extensions. IEEE Transactions on Information Theory 62(10), 5918-5937.

Har-Peled, S. (1998). On the expected complexity of random convex hulls. Technical Report 330/98, School of Mathematical Sciences, Tel Aviv University.

Hartline, J. D. (2017). Mechanism design and approximation. Book in preparation.

Hoare, C. A. R. (1962). Quicksort. The Computer Journal 5(1), 10-15.

Holland, P. W., K. Lasket, and S. Leinhardt (1983). Stochastic blockmodels: First steps. Social Networks 5(2), 109-137. 
Jerrum, M. (1992). Large cliques elude the Metropolis process. Random Structures and Algorithms 3(4), 347-359.

Johnson, D. S. (1973). Near-Optimal Bin Packing Algorithms. Ph. D. thesis, MIT.

Johnson, D. S., A. Demers, J. D. Ullman, M. R. Garey, and R. L. Graham (1974). Worst-case performance bounds for simple one-dimensional packing algorithms. SIAM Journal on Computing 3(4), 299-325.

Karp, R. M. (1977). Probabilistic analysis of partitioning algorithms for the traveling-salesman problem in the plane. Mathematics of Operations Research 2(3), 209-224.

Karp, R. M. and J. M. Steele (1985). Probabilistic analysis of heuristics. In E. L. Lawler, J. K. Lenstra, A. H. G. Rinnooy Kan, and D. B. Shmoys (Eds.), The Traveling Salesman Problem, Chapter 6, pp. 181-205. John Wiley \& Sons.

Knuth, D. E. (1998). The Art of Computer Programming: Sorting and Searching, Volume 3. Addison-Wesley. Second edition.

Kucera, L. (1995). Expected complexity of graph partitioning problems. Discrete Applied Mathematics 57(2-3), 193-212.

Matula, D. W. (1976). The largest clique size in a random graph. Technical Report 7608, Department of Computer Science, Southern Methodist University.

Mitchell, J. S. B. (1999). Guillotine subdivisions approximate polygonal subdivisions: A simple polynomial-time approximation scheme for geometric tsp, k-mst, and related problems. SIAM Journal on Computing 28(4), 1298-1309.

Mitzenmacher, M. and E. Upfal (2017). Probability and Computing. Cambridge. Second edition.

Rényi, A. and R. Sulanke (1963). Über die konvexe hülle von $n$ zugällig gewählten punkten. Zeitschrift für Wahrscheinlichkeitstheorie und Verwandte Gebiete 2, 75-84.

Roughgarden, T. (Ed.) (2020). Beyond the Worst-Case Analysis of Algorithms. Cambridge University Press.

Samuel-Cahn, E. (1984). Comparison of threshold stop rules and maximum for independent nonnegative random variables. Annals of Probability 12(4), 1213-1216.

\section{Exercises}

1. Extend the prophet inequality (Theorem 1.1) to the case in which there is no threshold $t$ with $q(t)=\frac{1}{2}$, where $q(t)$ is the probability that no prize meets the threshold.

[Hint: Define $t$ such that $\operatorname{Pr}\left[\pi_{i}>t\right.$ for all $\left.i\right] \leq \frac{1}{2} \leq \operatorname{Pr}\left[\pi_{i} \geq t\right.$ for all $\left.i\right]$. Show that at least one of the two corresponding strategies - either taking the first prize with value at least $t$, or the first with value exceeding $t$ - satisfies the requirement.]

2. The prophet inequality (Theorem 1.1) provides an approximation guarantee of $\frac{1}{2}$ relative to the expected prize value obtained by a prophet, which is at least (and possibly more than) the expected prize value obtained by an optimal online algorithm. Show by examples that the latter quantity can range from $50 \%$ to $100 \%$ of the former. 
3. Prove that for a bin packing instance consisting of 6 items with size $\frac{1}{2}+\epsilon, 6$ items with size $\frac{1}{4}+2 \epsilon, 6$ jobs with size $\frac{1}{4}+\epsilon$, and 12 items with size $\frac{1}{4}-2 \epsilon$, the first-fit decreasing algorithm uses 11 bins and an optimal solution uses 9 bins.

4. This exercise and the next outline a proof of Theorem 2.2. Divide the interval $[0,1]$ evenly into $n^{1 / 4}$ intervals, with $I_{j}$ denoting the subinterval $\left[\frac{j-1}{n^{1 / 4}}, \frac{j}{n^{1 / 4}}\right]$ for $j=1,2, \ldots, n^{1 / 4}$. Let $P_{j}$ denote the items with size in $I_{j}$.

(a) One version of the Chernoff bound states that, for every sequence $X_{1}, X_{2}, \ldots, X_{n}$ of Bernoulli (0-1) random variables with means $p_{1}, p_{2}, \ldots, p_{n}$ and every $\delta \in(0,1)$,

$$
\operatorname{Pr}[|X-\mu| \geq \delta \mu] \leq 2 e^{-\mu \delta^{2} / 3}
$$

where $X$ and $\mu$ denote $\sum_{i=1}^{n} X_{i}$ and $\sum_{i=1}^{n} p_{i}$, respectively. Use this bound to prove that

$$
\left|P_{j}\right| \in\left[n^{3 / 4}-\sqrt{n}, n^{3 / 4}+\sqrt{n}\right] \text { for all } j=1,2, \ldots, n^{1 / 4}
$$

with probability $1-o(1)$ as $n \rightarrow \infty$.

(b) Assuming (6), prove that the sum $\sum_{i=1}^{n} s_{i}$ is at least $\frac{1}{2} n-c_{1} n^{3 / 4}$ for some constant $c>0$. What does this imply about the number of bins used by an optimal solution?

(c) Assuming (6), prove that in the third step of the TM algorithm, every pair of items $i$ and $k-i+1$ fits in a single bin.

(d) Conclude that there is a constant $c_{2}>0$ such that, when property (6) holds, the TM algorithm uses at most $\frac{1}{2} n+c_{2} n^{3 / 4}=(1+o(1)) \cdot O P T$ bins, where OPT denotes the number of bins used by an optimal solution.

5. Prove Lemma 2.1.

6. Give an algorithm that, given a set $S$ of $n$ points from the square sorted by $x$-coordinate, computes the convex hull of $S$ in $O(n)$ time.

[Hint: compute the lower and upper parts of the convex hull separately.]

7. Prove that the convex hull of $n$ points drawn independently and uniformly at random from the unit square occupies a $1-O\left(\frac{\log n}{n}\right)$ fraction of the square.

8. Prove Lemma 3.2.

[Hint: Chop the unit square evenly into $n$ subsquares of side length $n^{-1 / 2}$, and each subsquare further into 9 mini-squares of side length $\frac{1}{3} \cdot n^{-1 / 2}$. For a given subsquare, what is the probability that the input includes one point from its center mini-square and none from the other 8 mini-squares?]

9. Another variation of the Chernoff bound states that, for every sequence $X_{1}, X_{2}, \ldots, X_{n}$ of Bernoulli (0-1) random variables with means $p_{1}, p_{2}, \ldots, p_{n}$ and every $t \geq 6 \mu$,

$$
\operatorname{Pr}[X \geq t] \leq 2^{-t}
$$

where $X$ and $\mu$ denote $\sum_{i=1}^{n} X_{i}$ and $\sum_{i=1}^{n} p_{i}$, respectively. Use this bound to prove Lemma 3.3.

10. Prove Lemma 3.4. 
11. Use the Chernoff bound from Exercise 4(a) to prove that, with probability approaching 1 as $n \rightarrow \infty$, every bisection of a random graph from $\mathcal{G}_{n, p}$ has $(1 \pm o(1)) \cdot \frac{n^{2}}{8}$ crossing edges.

12. Consider the planted bisection problem with parameters $p=c_{1}$ and $q=p-c_{2}$ for constants $c_{1}, c_{2}>0$. Consider the following simple combinatorial algorithm for recovering a planted bisection:

- Choose a vertex $v$ arbitrarily.

- Let $A$ denote the $\frac{n}{2}$ vertices that have the fewest common neighbors with $v$.

- Let $B$ denote the rest of the vertices (including $v$ ) and return $(A, B)$.

Prove that, with high probability over the random choice of $G$ (approaching 1 as $n \rightarrow \infty$ ), this algorithm exactly recovers the planted bisection.

[Hint: compute the expected number of common neighbors for pairs of vertices on the same and on different sides of the planted partition. Use the Chernoff bound.]

13. Consider the planted clique problem (Section 4.2) with planted clique size $k \geq c \log _{2} n$ for a sufficiently large constant $c$. Design an algorithm that runs in $n^{O(\log n)}$ time and, with probability $1-o(1)$ as $n \rightarrow \infty$, recovers the planted clique. 